\title{
Lorenz integrable system moves à la Poinsot
}

\author{
M. C. Nucci \\ Dipartimento di Matematica e Informatica \\ Università di Perugia, 06123 Perugia, Italy \\ e-mail: nucci@unipg.it
}

\begin{abstract}
A transformation is derived which takes the Lorenz integrable system into the wellknown Euler equations of a torque-free rigid body about a fixed point, i.e. the famous motion à la Poinsot. The proof is based on Lie group analysis applied to two thirdorder ordinary differential equations admitting the same two-dimensional Lie symmetry algebra. Lie's classification of two-dimensional symmetry algebras in the plane is used. If the same transformation is applied to the Lorenz system with any values of the parameters, then one obtains Euler equations of a rigid body about a fixed point subjected to a torsion depending on time and angular velocity. The numerical solution of this system yields a three-dimensional picture which looks like a "tornado" the crosssection of which has a butterfly-shape. Thus Lorenz's butterfly has been transformed into a tornado.
\end{abstract}

Keywords: rigid body, Lorenz system, Lie symmetry algebra

PACS numbers: 45.40.Cc, 47.27.Te, 02.20.Sv

Running title: Lorenz integrable system moves à la Poinsot 


\section{Introduction}

The motion of a heavy rigid body about a fixed point is one of the most famous problems of classical mechanics [1]. In 1750 Euler [2] derived the equations of motion, which now bear his name, and described what is nowadays known as the Euler-Poinsot case because of the geometrical description given by Poinsot about hundred years later [3]. It was Jacobi [4] who integrated this case by using the elliptic functions which he had developed (along with Legendre, Abel and Gauss [5]) and mastered [6] - we have translated this fundamental text into Italian and commented extensively [7].

More than 200 years later, in 1963, a paper was published [8] in which was presented a system of three ordinary differential equations. The author considered a hydrodynamical system developed by Rayleigh [9] and reduced it by applying a double Fourier series as in [10. Thus he obtained what nowadays is the famous Lorenz system [11]. Three parameters are part of the Lorenz system. For particular values of those parameters the Lorenz system can be integrated in closed form by means of Jacobi elliptic functions [12. We call this system the Lorenz integrable system.

In January 2001 the first Whiteman prize for notable exposition on the history of mathematics was awarded to Thomas Hawkins by the American Mathematical Society. In the citation, published in the Notices of AMS 48 416-417 (2001), one reads that Thomas Hawkins “... has written extensively on the history of Lie groups. In particular he has traced their origins to [Lie's] work in the 1870 s on differential equations ... the idée fixe guiding Lie's work was the development of a Galois theory of differential equations ... [Hawkins's book [13] highlights the fascinating interaction of geometry, analysis, mathematical physics, algebra and topology ...". Also Hawkins had established "the nature and extent of Jacobi's influence upon Lie" [14].

In the Introduction of his book [15] Olver wrote that "it is impossible to overestimate the importance of Lie's contribution to modern science and mathematics. Nevertheless anyone who is already familiar with [it] . . is perhaps surprised to know that its original inspirational 
source was the field of differential equations".

Lie's monumental work on transformation groups, [16, [17] and [18, and in particular contact transformations [19], led him to achieve his goal [20].

Many books have been dedicated to this subject and its generalizations ([21], [22], [23], [15], [24], 25], 26], 27], 28] and [29]).

Lie group analysis is indeed the most powerful tool to find the general solution of ordinary differential equations. Any known integration technique can be shown to be a particular case of a general integration method based on the derivation of the continuous group of symmetries admitted by the differential equation, i.e. the Lie symmetry algebra. In particular Bianchi's theorem ([30], [15]) states that, if an admitted $n$-dimensional solvable Lie symmetry algebra is found, then the general solution of the corresponding $n^{\text {th }}$-order system of ordinary differential equations can be obtained by quadratures. The admitted Lie symmetry algebra can be easily derived by a straightforward although lengthy procedure. As computer algebra software becomes widely used, the integration of systems of ordinary differential equations by means of Lie group analysis is becoming easier to perform. A major drawback of Lie's method is that it is useless when applied to systems of $n$ first-order equations, because they admit an infinite number of symmetries, and there is no systematic way to find even an one-dimensional Lie symmetry algebra, apart from trivial groups like translations in time admitted by autonomous systems. One may try to derive an admitted $n$-dimensional solvable Lie symmetry algebra by making an ansatz on the form of its generators.

However, in 31] we have remarked that any system of $n$ first-order equations could be transformed into an equivalent system where at least one of the equations is of second-order. Then the admitted Lie symmetry algebra is no longer infinite-dimensional, and nontrivial symmetries of the original system could be retrieved [31. This idea has been successfully applied in several instances ([31], 32, 33] and [34]). Also in [35] we have have shown that first integrals can be obtained by Lie group analysis even if the system under study does not come from a variational problem, i.e., without making use of Noether's theorem [36]. If 
we consider a system of first-order equations and, by eliminating one of the dependent variables, derive an equivalent system which has one equation of second-order, then Lie group analysis applied to that equivalent system yields the first integral(s) of the original system which do(es) not contain the eliminated dependent variable. Of course this requires that such first integrals exist. The procedure should be repeated as many times as there are dependent variables in order to find all such first integrals. The first integrals correspond to the characteristic curves of determining equations of parabolic type which are constructed by the method of Lie group analysis. We remark that interactive (not automatic) programs for calculating Lie symmetries such as [37] and 38] are more appropriate for performing this task.

We have briefly sketched three apparently unrelated subjects. In this paper we show that the Lorenz system and the Euler equations are actually related by means of Lie group analysis. In [39] we applied Lie group analysis to a third-order differential equation, which is equivalent to the Lorenz integrable system, and obtained a two-dimensional Lie symmetry algebra, which we then used to integrate the Lorenz integrable system in terms of Jacobi elliptic functions. Here we show that the same Lie symmetry algebra is admitted by a third-order differential equation which is equivalent to the Euler equations of a torque-free rigid body moving about a fixed point. Then a transformation is easily derived by which the Lorenz integrable system becomes the Euler equations of a torque-free rigid body moving about a fixed point. Thus, it can be stated that "the Lorenz integrable system moves à la Poinsot". If the same transformation is applied to the Lorenz system with any value of parameters, then one obtains the Euler equations of a rigid body moving about a fixed point and subjected to a torsion depending on time and angular velocity. The numerical solution of this system yields a three-dimensional picture which resembles a "tornado" the cross-section of which has a butterfly-shape. By means of our transformation Lorenz's butterfly becomes a tornado.

In the last section the relationship between Lie group analysis and first integrals [35] is ex- 
emplified by considering the Euler equations of a torque-free rigid body about a fixed point and the Lorenz integrable system.

\section{Butterflies and tornadoes}

Consider the Lorenz system [8]:

$$
\begin{aligned}
x^{\prime} & =\tilde{\sigma}(y-x), \\
y^{\prime} & =-x z+\tilde{r} x-y, \\
z^{\prime} & =x y-\tilde{b} z,
\end{aligned}
$$

where $\tilde{\sigma}, \tilde{b}$ and $\tilde{r}$ are parameters (a prime denotes differentiation with respect to $\tau$ ). This system can be reduced to a single third-order ordinary differential equation for $x$ [41, which admits a two-dimensional Lie symmetry algebra if $\tilde{\sigma}=1 / 2, \tilde{b}=1$ and $\tilde{r}=0$. System (11,3) becomes

$$
\begin{aligned}
x^{\prime} & =\frac{(y-x)}{2}, \\
y^{\prime} & =-x z-y, \\
z^{\prime} & =x y-z .
\end{aligned}
$$

The corresponding third-order equation is:

$$
2 x x^{\prime \prime \prime}-2 x^{\prime} x^{\prime \prime}+5 x x^{\prime \prime}-3 x^{2}+2 x^{3} x^{\prime}+3 x x^{\prime}+x^{4}+x^{2}=0,
$$

and admits a two-dimensional Lie symmetry algebra $L_{2}$ with basis:

$$
X_{1}=\partial_{\tau}, \quad X_{2}=e^{\tau / 2}\left(\partial_{\tau}-\frac{1}{2} x \partial_{x}\right)
$$

A basis of its differential invariants of-order $\leq 2$ is given by:

$$
\phi=\left(x^{\prime}+\frac{x}{2}\right) x^{-2}, \quad \psi=\left(x^{\prime \prime}+\frac{3}{2} x^{\prime}+\frac{x}{2}\right) x^{-3} .
$$

Equation (77) is reduced to the following first-order equation:

$$
\left(\psi-2 \phi^{2}\right) \frac{\mathrm{d} \psi}{\mathrm{d} \phi}=-2 \psi \phi-\phi
$$


which can be easily integrated [40] to give:

$$
\frac{1+4 \psi-4 \phi^{2}}{(1+2 \psi)^{2}}=c_{1},
$$

where $c_{1}$ is an arbitrary constant. Substitution of $x$ and its derivatives into (11) yields a second-order ordinary differential equation

$$
\frac{1+4\left(x^{\prime \prime}+\frac{3}{2} x^{\prime}+\frac{1}{2} x\right) x^{-3}-4\left(x^{\prime}+\frac{1}{2} x\right)^{2} x^{-4}}{\left(x^{3}+2 x^{\prime \prime}+3 x^{\prime}+x\right)^{2} x^{-6}}=c_{1},
$$

which admits the Lie symmetry algebra $L_{2}$. Lie's classification of two-dimensional algebras into four canonical types [20] allows us to integrate (12) by quadrature if we introduce the canonical variables:

$$
v=-2 e^{-\tau / 2}, \quad u=\frac{e^{-\tau / 2}}{x}
$$

which transform equation (12) into

$$
\frac{1+4\left(\frac{\mathrm{d} u}{\mathrm{~d} v}\right)^{2}-4 u \frac{\mathrm{d}^{2} u}{\mathrm{~d} v^{2}}}{\left[2 u \frac{\mathrm{d}^{2} u}{\mathrm{~d} v^{2}}-4\left(\frac{\mathrm{d} u}{\mathrm{~d} v}\right)^{2}-1\right]^{2}}=c_{1},
$$

and operators (8) into

$$
\bar{X}_{1}=\partial_{v}, \quad \bar{X}_{2}=v \partial_{v}+u \partial_{u} .
$$

Then the general solution of (14) can be easily derived [20] to be:

$$
\int\left(-c_{1} \mp 2 c_{2} u^{2}-c_{2}^{2} u^{4}\right)^{-1 / 2} \mathrm{~d} u= \pm \frac{v}{2 \sqrt{c_{1}}}+c_{3}
$$

with $c_{2}$ and $c_{3}$ arbitrary constants. This solution which involves an elliptic integral has already been obtained by Sen and Tabor [41] by means of a lengthier analysis.

The Euler equations describing the motion of a heavy rigid body about a fixed point with no torsion are

$$
\begin{aligned}
\dot{p} & =\frac{(B-C)}{A} q r, \\
\dot{q} & =\frac{(C-A)}{B} p r, \\
\dot{r} & =\frac{(A-B)}{C} p q,
\end{aligned}
$$


with $A, B$ and $C$ being the principal moments of inertia, and $p(t), q(t)$ and $r(t)$ the components of the angular velocity (a dot denotes differentiation with respect to $t$ ). This system can be reduced to a single third-order ordinary differential equation for, say, $p$, viz

$$
p \frac{\mathrm{d}^{3} p}{\mathrm{~d} t^{3}}-\frac{\mathrm{d} p}{\mathrm{~d} t} \frac{\mathrm{d}^{2} p}{\mathrm{~d} t^{2}}-\frac{4(C-A)(A-B)}{B C} p^{3} \frac{\mathrm{d} p}{\mathrm{~d} t}=0,
$$

which admits a two-dimensional Lie symmetry algebra $\mathcal{L}_{2}$ with basis:

$$
\Gamma_{1}=\partial_{t}, \quad \Gamma_{2}=t \partial_{t}-p \partial_{p}
$$

The two Lie symmetry algebras $L_{2}$ and $\mathcal{L}_{2}$ that we have found are actually the same, i.e. Type IV in Lie's classification [20]. Therefore they are linked by a transformation which takes $(\tau, x)$ into $(t, p)$. Prolongation to the second-order of the two equivalent Lie symmetry algebras yields a transformation which takes the system (17)-(19) into the system (44)-(6) as

$$
\begin{aligned}
\tau & =\log \left(\frac{4}{t^{2}}\right) \\
x & =\frac{p t}{2}, \\
y & =\frac{C-B}{2 A} q r t^{2}, \\
z & =\frac{C-B}{2 A}\left[\frac{(C-A)}{B} r^{2}+\frac{(A-B)}{C} q^{2}\right] t^{2},
\end{aligned}
$$

with the following condition on the momenta of inertia

$$
\frac{(A-B)(A-C)}{B C}=\frac{1}{4}
$$

A slightly more general condition could have been considered if one replaces $1 / 4$ with $k / 4(k$ an arbitrary parameter). If one derives $B$ from (23) by assuming $A-C>0$ and $4 A-3 C>0$, i.e.

$$
B=\frac{4 A(A-C)}{4 A-3 C}
$$

then the transformation (22) turns into the following

$$
\tau=\log \left(\frac{4}{t^{2}}\right)
$$




$$
\begin{aligned}
& x=\frac{p t}{2} \\
& y=-\frac{(2 A-C)(2 A-3 C)}{2 A(4 A-3 C)} q r t^{2}, \\
& z=-\frac{(2 A-C)(2 A-3 C)\left[4 A^{2} q^{2}-(4 A-3 C)^{2} r^{2}\right]}{8 A^{2}(4 A-3 C)^{2}} t^{2},
\end{aligned}
$$

and the system (17)-(19) assumes the form

$$
\begin{aligned}
\dot{p} & =\frac{(2 A-C)(2 A-3 C)}{A(4 A-3 C)} q r, \\
\dot{q} & =\frac{3 C-4 A}{4 A} p r, \\
\dot{r} & =\frac{A}{4 A-3 C} p q .
\end{aligned}
$$

We also derive the inverse transformation, i.e.

$$
\begin{aligned}
t & =2 e^{-\tau / 2}, \\
p & =x e^{\tau / 2}, \\
q & =-\frac{(4 A-3 C) y e^{\tau / 2}}{2 \sqrt{(2 A-C)(2 A-3 C)\left(\sqrt{y^{2}+z^{2}}+z\right)}}, \\
r & =\frac{A e^{\tau / 2} \sqrt{\sqrt{y^{2}+z^{2}}+z}}{\sqrt{(2 A-C)(2 A-3 C)}},
\end{aligned}
$$

which takes the system (44)-(6) into the system (17)-(19) after substituting $B$ as in (24).

If one applies the transformation (29) to the general Lorenz system (10)-(3), then the following equations are obtained

$$
\begin{aligned}
\dot{p}= & \frac{2(2 A-C)(2 A-3 C) \tilde{\sigma}}{A(4 A-3 C)} q r+(2 \tilde{\sigma}-1) \frac{p}{t}, \\
\dot{q}= & \frac{3 C-4 A}{4 A} p r+(\tilde{b}-1) \frac{4 A^{2} q^{2}-(4 A-3 C)^{2} r^{2}}{4 A^{2} q^{2}+(4 A-3 C)^{2} r^{2}} \frac{q}{t} \\
& +\tilde{r} \frac{2(4 A-3 C)^{3} A}{(2 A-C)(2 A-3 C)\left[4 A^{2} q^{2}+(4 A-3 C)^{2} r^{2}\right]} \frac{p r}{t^{2}}, \\
\dot{r}= & \frac{A}{4 A-3 C} p q-(\tilde{b}-1) \frac{4 A^{2} q^{2}-(4 A-3 C)^{2} r^{2}}{4 A^{2} q^{2}+(4 A-3 C)^{2} r^{2}} \frac{r}{t} \\
& +\tilde{r} \frac{8(4 A-3 C) A^{3}}{(2 A-C)(2 A-3 C)\left[4 A^{2} q^{2}+(4 A-3 C)^{2} r^{2}\right]} \frac{p q}{t^{2}} .
\end{aligned}
$$


They can be interpreted as the Euler equations of a rigid body moving about a fixed point and subjected to a torsion which depends on time $t$ and angular velocity $(p, q, r)$ in the body-frame reference. Also the momenta of inertia are linked by relation (24).

To our knowledge such a system has never been described.

If we use Maple $\mathrm{V}$ in order to draw a three-dimensional plot of system (301)-(32), then a "tornado", the cross-section of which resembles a butterfly, is obtained (FIG. 1). The usual values for the Lorenz parameters, $\tilde{\sigma}=10, \tilde{b}=8 / 3, \tilde{r}=28$, are imposed. Also we assume $A=2$ and $C=1$. We consider $t$ as it varies in the interval [2,0.015], which corresponds to $\tau \in[0,9.8]$ approximately. The step size used is 0.00005 .

The butterfly-shape curve is better seen in FIG. 2, which shows the two-dimensional plot of $p$ versus $q$.

A clearer view of the "tornado" is given in FIG. 3, which shows the two-dimensional plot of $r$ versus $q$. Another view can be found in FIG. 4 which shows the two-dimensional plot of $r$ versus $p$.

In FIG. 5 and 6 the plot of $p$ versus $t$ is given for two different but close initial values (1 and 1.01). For relatively large values of $t$, say $t \in[2,0.5]$, the solutions are the same (FIG. 5). For small $t$, say $t \in[0.05,0.015]$, a dramatic difference appears (FIG. 6).

In [42] it was found that one could explicitly derive a first integral of the Lorenz system (11)(3) in six different instances which correspond to particular values of the Lorenz parameters. It is a trivial task to apply those findings to system (30)-(32) by using the transformation (22). The following is the list of the six cases with a relative first integral $F$ for the system (301)- (32) .

(1) $\tilde{b}=2 \tilde{\sigma}, \tilde{r}$ arbitrary

$$
F=4^{2 \tilde{\sigma}-1} t^{2(1-2 \tilde{\sigma})}\left(p^{2}+\frac{\left[4 A^{2} q^{2}-(4 A-3 C)^{2} r^{2}\right](2 A-C)(2 A-3 C) \tilde{\sigma}}{A^{2}(4 A-3 C)^{2}}\right) .
$$

(2) $\tilde{b}=0, \tilde{\sigma}=\frac{1}{3}, \tilde{r}$ arbitrary

$$
F=4^{1 / 3} t^{-2 / 3}\left(-p^{2} \tilde{r}-2 \frac{(2 A-C)(2 A-3 C)}{3 A(4 A-3 C)} p q r t-\frac{3}{16} p^{4} t^{2}\right.
$$




$$
\left.-\frac{\left(4 A^{2} q^{2}-(4 A-3 C)^{2} r^{2}\right)(2 A-C)(2 A-3 C)}{8 A^{2}(4 A-3 C)^{2}} p^{2} t^{2}+\frac{(2 A-C)^{2}(2 A-3 C)^{2}}{3 A^{2}(4 A-3 C)^{2}} q^{2} r^{2} t^{2}\right) .
$$

(3) $\tilde{b}=1, \tilde{r}=0, \tilde{\sigma}$ arbitrary

$$
F=\frac{\left(4 A^{2} q^{2}+(4 A-3 C)^{2} r^{2}\right)^{2}(2 A-C)^{2}(2 A-3 C)^{2}}{4 A^{4}(4 A-3 C)^{4}} .
$$

(4) $\tilde{b}=4, \tilde{\sigma}=1, \tilde{r}$ arbitrary

$$
\begin{aligned}
F= & 4 t^{-6}\left(16 p^{2} \tilde{r}+32 \tilde{r} \frac{\left(4 A^{2} q^{2}-(4 A-3 C)^{2} r^{2}\right)(2 A-C)(2 A-3 C)}{A^{2}(4 A-3 C)^{2}}-p^{4} t^{2}\right. \\
& -2 \frac{\left(4 A^{2} q^{2}-(4 A-3 C)^{2} r^{2}\right)(2 A-C)(2 A-3 C)}{A^{2}(4 A-3 C)^{2}} p^{2} t^{2}+32 \frac{(2 A-C)(2 A-3 C)}{A(4 A-3 C)} p q r t \\
& \left.+16 \frac{(2 A-C)^{2}(2 A-3 C)^{2}}{A^{2}(4 A-3 C)^{2}} q^{2} r^{2} t^{2}-32 \frac{\left(4 A^{2} q^{2}-(4 A-3 C)^{2} r^{2}\right)(2 A-C)(2 A-3 C)}{A^{2}(4 A-3 C)^{2}}\right) .
\end{aligned}
$$

(5) $\tilde{b}=1, \tilde{\sigma}=1, \tilde{r}$ arbitrary

$$
F=-\frac{4}{t^{2}} p^{2} \tilde{r}+\frac{\left(4 A^{2} q^{2}+(4 A-3 C)^{2} r^{2}\right)^{2}(2 A-C)^{2}(2 A-3 C)^{2}}{4 A^{4}(4 A-3 C)^{4}} .
$$

(6) $\tilde{b}=6 \tilde{\sigma}-2, \tilde{r}=2 \tilde{\sigma}-1$

$$
\begin{aligned}
F= & 4^{4 \tilde{\sigma}} t^{2(1-4 \tilde{\sigma})}\left(-\frac{p^{4} t^{2}}{64 \tilde{\sigma}}-\tilde{\sigma} \frac{\left(4 A^{2} q^{2}-(4 A-3 C)^{2} r^{2}\right)(2 A-C)(2 A-3 C)}{32 A^{2}(4 A-3 C)^{2}} p^{2} t^{2}\right. \\
& \left.+\frac{(2 \tilde{\sigma}-1)^{2}}{4 \tilde{\sigma}} p^{2}+\frac{(2 A-C)(2 A-3 C)(2 \tilde{\sigma}-1)}{2 A(4 A-3 C)} p q r t+\frac{(2 A-C)^{2}(2 A-3 C)^{2} \tilde{\sigma}}{4 A^{2}(4 A-3 C)^{2}} q^{2} r^{2} t^{2}\right) .
\end{aligned}
$$

\section{$3 \quad$ Lie group analysis and first integrals}

In [35] we showed the application of Lie group analysis in order to obtain first integrals with at least one missing variable. Consider Euler equations (17)-(19). There exist two wellknown first integrals, i.e. the conservation of kinetic energy and the conservation of angular momentum:

$$
\begin{gathered}
A p^{2}+B q^{2}+C r^{2}=I_{1}, \\
A^{2} p^{2}+B^{2} q^{2}+C^{2} r^{2}=I_{2} .
\end{gathered}
$$


We derive $p$ from (19) (the method works independently of the chosen equation) as:

$$
p=\frac{C \dot{r}}{(A-B) q}
$$

in order to obtain the following two differential equations in $q$ and $r$, one of first-order and one second-order, respectively:

$$
\begin{aligned}
\dot{q} & =\frac{C\left(C_{A}\right) r \dot{r}}{B(A-B) q} \\
\ddot{r} & =\frac{C(C-A)}{B(A-B) q^{2}} r \dot{r}^{2}+\frac{(B-C)(A-B)}{A C} r q^{2} .
\end{aligned}
$$

When Lie group analysis of the system (35)-(36) is performed, a linear partial differential equation of parabolic structure is generated. Its characteristic curve is given by

$$
B(A-B) q^{2}+C(A-C) r^{2}
$$

which is a combination of the two first integrals (33) and (34). Consequently we introduce the new dependent variable $s$ such that

$$
q=\sqrt{\frac{C(C-A) r^{2}+s}{B(A-B)}}
$$

in order to obtain the following system

$$
\begin{aligned}
\dot{s} & =0, \\
\ddot{r} & =\frac{C(C-A) r \dot{r}^{2}}{C(C-A) r^{2}+s}+\frac{(C-A)(B-C) r^{3}}{A B}+\frac{(B-C) s r}{A B C} .
\end{aligned}
$$

Equation (39) admits an eight-dimensional Lie symmetry algebra (i.e., it is linearizable) if either $A=C$ or $B=C$, i.e. the case of the torque-free Lagrange top (uniform precession). If either $q$ or $r$, one at a time, is eliminated from system (17)-(19), then a similar result is

obtained, i.e. the other two combinations of the first integrals (33) and (34). Indeed the elimination of $q$ yields the first integral

$$
A(A-B) p^{2}+C(B-C) r^{2},
$$


and the elimination of $r$ yields the first integral

$$
B(B-C) q^{2}+A(A-C) p^{2}
$$

If the same method is applied to the Lorenz integrable system (4)-(6), then the following two first integrals are obtained

$$
\begin{gathered}
\left(y^{2}+z^{2}\right) e^{2 \tau} \\
\left(z-x^{2}\right) e^{\tau}
\end{gathered}
$$

Those first integrals were found in [12] by using the Painlevé analysis.

From (6) we have

$$
x=\frac{z^{\prime}+z}{y}
$$

in order to obtain the following two differential equations in $y$ and $z$, one of first-order and one second-order, respectively:

$$
\begin{aligned}
y^{\prime} & =-\frac{y^{2}+z^{2}+z z^{\prime}}{y} \\
z^{\prime \prime} & =\frac{y^{4}-3 y^{2} z-5 y^{2} z^{\prime}-2 z^{3}-4 z^{2} z^{\prime}-2 z z^{\prime 2}}{2 y^{2}} .
\end{aligned}
$$

When the Lie group analysis of the system (42)-(43) is performed, a linear partial differential equation of parabolic structure is generated. Its characteristic curve is given by

$$
y^{2}+z^{2}
$$

Consequently we introduce the new dependent variable $Y$ such that

$$
y=\sqrt{Y-z^{2}}
$$

in order to obtain the following system

$$
\begin{aligned}
Y^{\prime} & =-2 Y \\
z^{\prime \prime} & =\frac{Y^{2}-2 Y z^{2}-3 Y z-5 Y z^{\prime}+z^{4}+z^{3}+z^{2} z^{\prime}-2 z z^{\prime 2}}{2\left(Y-z^{2}\right)} .
\end{aligned}
$$

The first equation can be easily integrated to yield the first integral (40).

If we eliminate $y$ from system (44)-(6), a similar procedure provides the first integral (41). 


\section{References}

[1] Golubev, V. V., Lectures on Integration of the Equations of Motions of a Rigid Body about a Fixed Point (Moscow: State Publishing House of Theoretical Technical Literature, 1953) (English translation published by the Israel Program for Scientific Literature in 1960).

[2] Euler, L., Theoria motus corporum solidorum seu rigidorum ex primis nostrae cognitionis principiis stabilita et ad omnes motus, qui in huiusmodi corpora cadere possunt, accommodata (Rostochii et Gryphiswaldiae: Litteris et Impensis A. F. Röse, 1765).

[3] Poinsot, L., "Théorie nouvelle de la rotations des corps," J. Math. Pures Appl. Sér I, 9-129 and 289-336 (1851).

[4] Jacobi, K. G. J., "Sur la rotation d'un corps," J. Reine Angew. Math. 39, 293-350 (1850).

[5] Natucci, A., "Le origini della teoria delle funzioni ellittiche (Nel I centenario della morte di Carlo Gustavo Jacobi 18 Febbraio 1851)," Atti Accad. Ligure 9, 40-54 (1953).

[6] Jacobi, K. G. J., Fundamenta Nova Theoriae Functionum Ellipticarum (Regiomonti: Fratrum Borntraeger, 1829).

[7] Rosati, L., Nucci, and M. C., Mezzanotte, F., "Edizione italiana e rilettura dei FUNDAMENTA NOVA di K. G. J. Jacobi a 150 anni dalla morte (18 Febbraio 1851 - 18 Febbraio 2001). Preprint RT n. 2001-4," Dipartimento di Matematica e Informatica, Università di Perugia (2001).

[8] Lorenz, E. N., "Deterministic nonperiodic flow," J. Atmos. Sci. 20, 130-141 (1963).

[9] Rayleigh, Lord, "On convective currents in a horizontal layer of fluid when the higher temperature is on the under side," Phil. Mag. 32, 529-546 (1916). 
[10] Saltzman, B., "Finite amplitude free convection as an initial value problem," J. Atmos. Sci. 19, 329-341 (1962).

[11] Dalmedico, A. D., "History and epistemology of models: meteorology (1946-1963) as a case study," Arch. His. Exact Sciences 55, 395-422 (2001).

[12] Segur, H., "Solitons and the Inverse Scattering Transform," Lect. Int. School Phys. 'Enrico Fermi', Varenna, Italy, 7-19 July (1980) Published in: Topics in Ocean-Physics, edited by A. R. Osborne and P. Malanotte Rizzoli (Amsterdam: North-Holland 1982), pp. $235-277$.

[13] Hawkins T., The Emergence of the Theory of Lie Groups: An Essay in the History of Mathematics 1869-1926 (New York: Springer-Verlag, 2000).

[14] Hawkins, T., "Jacobi and the birth of Lie's theory of groups," Arch. Hist. Exact Sciences 42, 187-278 (1991).

[15] Olver, P. J., Applications of Lie Groups to Differential Equations (Berlin: SpringerVerlag, 1986).

[16] Lie, S., Theorie der Transformationsgruppen. Erster Abschnitt (Leipzig: Teubner, 1888).

[17] Lie, S., Theorie der Transformationsgruppen. Zweiter Abschnitt (Leipzig: Teubner, 1890).

[18] Lie, S., Theorie der Transformationsgruppen. Dritter und Letzter Abschnitt (Leipzig: Teubner, 1893).

[19] Lie, S., Geometrie der Berührungstransformationen (Leipzig: Teubner, 1896).

[20] Lie, S., Vorlesungen über Differentialgleichungen mit Bekannten Infinitesimalen Transformationen (Leipzig: Teubner, 1912). 
[21] Ames, W. F., Nonlinear Partial Differential Equations in Engineering, Vol. 2 (New York: Academic Press, 1972).

[22] Bluman, G. W. and Cole, J. D., Similarity Methods for Differential Equations (Berlin: Springer-Verlag, 1974).

[23] Ovsjannikov, L. V., Group Analysis of Differential Equations (New York: Academic Press, 1982).

[24] Bluman, G. W. and Kumei, S., Symmetries and Differential Equations (Berlin: Springer-Verlag, 1989).

[25] Rogers, C. and Ames, W. F., Nonlinear Boundary Value Problems in Science and Engineering (New York: Academic Press, 1989).

[26] Stephani, H., Differential Equations. Their Solution Using Symmetries (Cambridge: Cambridge University Press, 1989).

[27] Hill, J. M., Differential Equations and Group Methods for Scientists and Engineers (Boca Raton: CRC Press, 1992).

[28] Ibragimov, N. H. (ed.), CRC Handbook of Lie Group Analysis of Differential Equations, Vol. I: Symmetries, Exact Solutions, and Conservation Laws (Boca Raton: CRC Press, 1994).

[29] Ibragimov, N. H. (ed.), CRC Handbook of Lie Group Analysis of Differential Equations, Vol. II: Applications in Engineering and Physical Sciences (Boca Raton: CRC Press, 1995).

[30] Bianchi, L., Lezioni sulla Teoria dei Gruppi Continui Finiti di Trasformazioni (Pisa: Enrico Spoerri Editore, 1918).

[31] Nucci, M. C., "The complete Kepler group can be derived by Lie group analysis," J. Math. Phys. 37, 1772-1775 (1996). 
[32] Torrisi, V. and Nucci, M. C., "Application of Lie group analysis to a mathematical model which describes HIV transmission," in The Geometrical Study of Differential Equations, edited by J. A. Leslie and T. P. Hobart (Providence: A.M.S., 2001), pp. 31-40.

[33] Nucci, M. C. and Leach, P. G. L., "The harmony in the Kepler and related problems," J. Math. Phys. 42, 746-764 (2001).

[34] Nucci, M. C. and Leach, P. G. L., "The essential harmony of the classical equations of mathematical physics," Preprint RT n. 2001-12, Dipartimento di Matematica e Informatica, Università di Perugia (2001).

[35] Marcelli, M. and Nucci, M. C., "Lie point symmetries and first integrals: the Kowalevsky top," J. Math. Phys. 44, 2111-2132 (2003).

[36] Noether, E., "Invariante Variationsprobleme". Nach König Gesell Wissen Göttingen Math-phys Kl, 235-257 (1918).

[37] Nucci, M. C., "Interactive REDUCE programs for calculating classical, non-classical and Lie-Bäcklund symmetries of differential equations," Preprint Georgia Tech Math 062090-051 (1990)

[38] Nucci, M. C., "Interactive REDUCE programs for calculating Lie point, non-classical, Lie-Bäcklund, and approximate symmetries of differential equations: manual and floppy disk," in CRC Handbook of Lie Group Analysis of Differential Equations. Vol. 3: New Trends, edited by N. H. Ibragimov (Boca Raton: CRC Press, 1996), pp. 415-481.

[39] Nucci, M. C., "The role of symmetries in solving differential equations," Mathl. Comput. Modelling 25, 181-193 (1997).

[40] Murphy, G. M., Ordinary Differential Equations and Their Solutions (Princeton: Van Nostrand, 1960). 
[41] Sen, T. and Tabor, M., "Lie symmetries of the Lorenz model," Physica D 44, 313-339 (1990).

[42] Kuś, M., "Integrals of motions for the Lorenz system," J. Phys. A: Math. Gen. 16, L689-L691 (1983). 


\section{Plot: figrigid01.eps}

Figure 1: The 3-dim plot of the "tornado".

\section{Plot: figrigid05.eps}

Figure 2: The 2-dim plot of $p$ vs. $q$. 


\section{Plot: figrigid06.eps}

Figure 3: The 2-dim plot of $r$ vs. $q$.

\section{Plot: rigids04.eps}

Figure 4: The 2-dim plot of $r$ vs. $p$. 


\section{Plot: rigids03.eps}

Figure 5: Varying the initial condition of $p$ in $t=2$ by 0.01 . The two plots of $p(t), t \in[2,0.5]$ are indistinguishable.

\section{Plot: fig6.eps}

Figure 6: Varying the initial condition of $p$ in $t=2$ by 0.01 . There are two different plots of $p(t), t \in[0.05,0.015]:$ the darker line represents the plot of $p(t)$ with initial condition $p=1$ in $t=2$, and the lighter line the plot of $p(t)$ with initial condition $p=1.01$ in $t=2$. 